\title{
Effets de la Politique Fiscale sur la Croissance Economique: Une Analyse sur Donnees de Panel Appliquee a Six Pays de L'Uemoa
}

\author{
Assi Tano Maxime, \\ Oulai Sieni Toussaint, \\ Département des Sciences Economiques, \\ Université Peleforo Gon Coulibay, Korhogo, Côte d'Ivoire
}

Doi:10.19044/esj.2019.v15n28p91～URL:http://dx.doi.org/10.19044/esj.2019.v15n28p91

\section{Résumé}

Dans cet article, nous étudions sur la base d'un modèle de croissance endogène, l'impact du niveau d'imposition du bénéfice des sociétés sur la croissance. L'outil économétrique privilégié est le modèle de transition lisse de panel (PSTR) fondé sur la composition de la structure fiscale. L'étude porte sur la période 1970-2016 et concerne six (6) pays de l'Union Economique et Monétaire Ouest Africaine (UEMOA). Les résultats obtenus révèlent que l'imposition des bénéfices des sociétés influence positivement l'économie lorsque le taux d'imposition retardé d'une période est inférieur à 12,7\%. Dans ce cas, la politique fiscale est efficace. Par contre, lorsque le niveau d'imposition des bénéfices des sociétés est supérieur au seuil critique, l'impact de la politique d'imposition sur la croissance devient négatif. Pour ce faire, nous recommandons un système fiscal moins distorsif, disproportionné, ciblé et démocratique.

Mots clés: Fiscalité, Impôt sur le bénéfice des sociétés, Croissance économique, Côte d'Ivoire, PSTR 


\title{
Effects of Tax Policy on Economic Growth: An Analysis on Panel Data Applied to Six Waemu Countries
}

\author{
Assi Tano Maxime, \\ Oulai Sieni Toussaint, \\ Département des Sciences Economiques, \\ Université Peleforo Gon Coulibay, Korhogo, Côte d'Ivoire
}

\begin{abstract}
In this paper, we study on the basis of an endogenous growth model, the impact of tax rates on profits on growth for six (6) countries of UEMOA, for the period 1970-2016. The preferred econometric tool is the Smooth Panel Transition Model (PSTR) based on the composition of the tax structure. The results show that tax of profits positively influences the economy when the tax rate delayed by one period is less than $12.7 \%$. In this case, the tax policy is effective. On the other hand, for tax of profits levels above the critical threshold, the impact of the corporate income tax policy on growth becomes negative. To achieve this, we would recommend a tax system that is less distortive, disproportionate, targeted and democratic.
\end{abstract}

Keywords: Taxation, Tax of profits, Growth, Ivory Coast, PST

\section{Introduction}

La politique fiscale désigne l'ensemble des mesures réglementaires et administratives (outils et mécanismes) permettant aux autorités politiques de collecter les impôts et taxes en vue de financer la production des biens collectifs et d'en assurer la redistribution (Avom, 2011). Vu sous cet angle, la fiscalité est à la fois une contribution à la croissance économique mais aussi un coût et donc susceptible d'affecter le profit des entreprises (Knuutinen, 2014).

Ainsi, si la mobilisation des recettes fiscales a effectivement un impact plutôt positif sur la croissance, cet impact est mitigé dès lors qu'on considère la structure du système fiscal, dont les variations contribuent à des divergences des performances économiques.

La littérature économique propose au moins deux approches : celle des partisans de libéralisme économique où l'impôt doit être le plus neuter possible et ne pas déstructurer le comportement des agents économiques et 
celle des tenants de l'interventionniste pigouvienne qui s'attache à corriger les défaillances du marché.

Selon la première approche, toute introduction d'un système fiscal dans une économie est porteuse d'imperfections du fait des distorsions produites dans le fonctionnement des marchés. Par exemple, l'application d'un taux de taxation au prix d'un bien peut modifier le signal véhiculé par ce prix, conduisant ainsi à la réduction de la demande pour ce bien et la diminution de la taille du marché.

En effet, le poids des prélèvements fiscaux peut obvier les externalités positives des recettes fiscales sur la croissance par une perte d'attractivité auprès des investisseurs nationaux et étrangers (Giménez, 2015), avec un impact négatif sur l'emploi. Il affecte aussi les incitations des agents à prendre des risques et à innover (Sioncke, 2017) et décourage les activités touristiques (Stiglitz, 2016).

De même, une fiscalité pesante engendre une perte de revenus pour l'Etat en faisant naître des comportements de fraude ou d'évasion fiscale (Dowling, 2014). L'optimalité de la pression fiscale a été illustrée par la courbe de Laffer. Cette courbe en « cloche » traduit l'idée qu'il existe un niveau optimal de taxation au delà duquel le produit de l'impôt diminue. Autrement dit « trop d'impôts tue l'impôt ».

Si le poids des prélèvements fiscaux entrave le développement des activités économiques, des taux d'imposition trop bas ne fournissent pas suffisamment de recettes fiscales, ce qui peut également provoquer un ralentissement économique. Ainsi, la seconde approche considère la fiscalité comme un instrument complémentaire à la réduction des dépenses à la disposition des gouvernements pour résorber les déséquilibres budgétaires. Le rôle de la fiscalité ne se limite pas à trouver des ressources budgétaires. Conformément à la typologie musgravienne de l'intervention publique, la fiscalité joue un rôle à la fois dans l'allocation des ressources, leur stabilité et leur redistribution.

De ce fait, les vertus de la fiscalité s'apprécient essentiellement en terme d'augmentation des ressources publiques (Minea \& Villieu, 2011); de réduction des inégalités (Duncan \& Peter, 2012) ; de moteur de l'investissement privé (Stiglitz, 2016) et de promotion de la croissance par tête (OCDE, 2012). Aussi, la fiscalité permet des créer des incitations pour soutenir la croissance et l'emploi (Stoilova, 2017) et elle contribue à la sauvegarde de l'environnement.

L'objet du présent article est de déterminer, sur la base d'un modèle de croissance endogène, l'impact du niveau d'imposition du bénéfice des sociétés sur la croissance pour six (6) pays de l'Union Economique et Monétaire Ouest Africaine (Uemoa), à savoir la Côte d'Ivoire, le Burkina Faso, le Sénégal, le Niger, le Bénin et le Togo pour la période 1970-2016. Le lien entre la 
croissance et le taux d'imposition est caractérisé par la possibilité d'une transition lisse entre deux régimes, à l'aide du modèle PSTR de González, Teräsvirta et Van Dijk (2005), révisé en 2017 (CREATES Research Paper 2017-36).

Depuis quelques décennies, l'étude de l'impact de la fiscalité sur la croissance suscite un intérêt particulier au sein de cette zone. Au moins deux raisons peuvent expliquer l'intérêt porté à cette étude. La première concerne la mobilisation des ressources nécessaires au financement du développement (Avom, 2011) qui constitue l'un des problèmes majeurs auxquels les pays de l'Afrique au Sud du Sahara font face. La seconde porte sur l'instabilité des recettes fiscales du fait de l'importante des variations dans le temps du taux de pression fiscale (Oura, 2012).

Nous présentons d'abord la revue de la littérature (II). Vient ensuite la méthodologie adoptée (III) et nous terminons enfin par la présentation de résultats (IV) et la conclusion (V).

\section{Revue de la littérature}

Si les résultats théoriques se rapportant à l'effet de la fiscalité sur la croissance sont nuancés, les évidences empiriques le sont encore davantage. En effet, sur la base d'un panel de 150 observations portant sur 10 pays de l'Afrique Centrale, Avom (2011) construit des indicateurs montrant un effet positif sur la croissance pour des niveaux plus bas de recettes fiscales. Et, à des niveaux plus élevés de taxes sur le revenu, l'effet devient ambigu.

De même, à l'aide d'un modèle de croissance endogène, Keho (2010) montre dans le cas de la Côte d'Ivoire, l'existence de relations de long terme entre les variables fiscales et le PIB et ses composantes. A court terme, par contre, l'auteur trouve que certains impôts réduisent la croissance économique.

Des résultats similaires ont été obtenus par l'OCDE (2009) en précisant que les impôts sur les sociétés sont le type d'impôt le plus nuisible à la croissance.

Beaucoup d'études montrent qu'une réduction de $1 \%$ de l'impôt sur les sociétés peut entraîner une augmentation du PIB de 0,1 à 0,6 \% (Gemmel, Kneller \& Sanz, 2011 ; Barro \& Redlick, 2011 ; Mertens \& Ravn, 2011). Barro (1990) généralise ces résultats en soutenant qu'une élévation de la fiscalité réduit le rendement marginal du capital privé, défavorable à la croissance.

Certains auteurs ont tiré la conclusion de l'existence d'un effet non linéaire de la fiscalité sur la croissance. Les résultats de leurs travaux montrent un faible impact sur la croissance des faibles taux d'imposition. Mais à mesure que les taux d'imposition augmentent, l'impact sur la croissance devient négatif (Jaimovich \& Rebelo, 2012).

D'autres par contre ne trouvent aucune corrélation entre les taux d'imposition et les taux de croissance. C'est le cas de Piketty, Saez \& 
Stantcheva (2014) qui souligne l'absence de corrélation entre les taux de croissance et les changements dans les taux marginaux d'imposition du revenu mis en œuvre dans les pays de l'OCDE depuis 1975.

Des résultats semblables ont été obtenus par Romer et Romer (2010) dans une étude portant sur l'impact de 104 modifications fiscales aux Etats- Unis. Ces auteurs montrent qu'une augmentation de $1 \%$ de l'impôt fédéral entraîne une baisse de $3 \%$ de la production après deux ans alors que des changements permanents de la fiscalité ne produit aucun impact sur le taux de croissance à long terme.

\section{Méthodologie}

L'objet du présent article est de déterminer, sur la base d'un modèle de croissance endogène, l'impact du niveau d'imposition du bénéfice des sociétés sur la croissance pour six (6) pays de l'Union Economique et Monétaire Ouest Africaine (Uemoa), à savoir la Côte d'Ivoire, le Burkina Faso, le Sénégal, le Niger, le Bénin et le Togo pour la période 1970-2016. Le lien entre la croissance et le taux d'imposition est caractérisé par la possibilité d'une transition lisse entre deux régimes, à l'aide du modèle PSTR de González, Teräsvirta et Van Dijk (2005), révisé en 2017 (CREATES Research Paper 2017-36).

Nous faisons l'hypothèse que l'impact sur la croissance de l'impôt sur le bénéfice des sociétés est positif. Mais à mesure que les taux d'imposition augmentent, l'efficacité de la politique fiscale est réduite ce qui nuit à la croissance économique.

\section{Cadre d'analyse théorique}

Nous prenons appui sur les travaux de Ferede \& Dahlby (2012) qui se sont basés sur le modèle initialement élaboré par Barro \& Sala-i-Martin (1992). Mais, à la différence de Ferede \& Dahlby qui ont montré à partir d'un modèle de croissance néoclassique, les effets des taux d'imposition sur la croissance et l'investissement privé, nous réduisons ce modèle et l'adaptons au cas de l'Uemoa en nous limitant seulement à l'impact sur la croissance dans le cadre d'un modèle de croissance endogène à la Barro (1990).

Ainsi, nous considérons une petite économie ouverte dotée d'une mobilité parfaite des capitaux. Cette économie n'a qu'un impact limité sur le taux d'intérêt international et n'a aucune restriction d'accès aux marchés financiers internationaux. Elle est composée de deux types d'agents économiques : un agent privé et un agent public.

L'agent privé, une firme représentative, agit sur un marché parfaitement concurrentiel et prend des décisions d'investissement privé. Contrairement à Ferede \& Dahlby (2012), la firme utilise à chaque période du 
capital privé, du travail et du capital public comme facteurs de production. Sa fonction de production est une fonction de type Cobb-Douglas telle que :

$$
Y_{t}=A K_{t}\left(G_{t 1}^{p} L_{t}\right)
$$

avec :

- t, la dimension temporelle;

- A, un choc technologique exogène ;

- , l'élasticité de substitution du produit aux facteurs capital public et travail

où $0 \prec \alpha \prec 1$, et $\quad$ son élasticité de substitution au capital privé, où $0 \prec \beta \prec 1$;

- $K_{t}$, le capital privé ;

- $L_{t}$, le facteur travail ;

- $G_{t 1}^{p}$, le capital public. Il correspond aux dépenses publiques de formation et de santé reçues par les agents et susceptibles d'accroître l'efficacité du travail. Le montant des dépenses publiques est décidé et pris en charge par l'État. Les dépenses de formation et de santé des travailleurs $\left(G_{t 1}^{p}\right)$ sont supposées être des externalités.

En raison de ces externalités, nous supposons croissants les rendements d'échelle de la fonction de production.

A chaque période, l'entreprise perçoit le produit des ventes $\left(p_{t} Y_{t}\right)$ où $p_{t}$ est le prix du bien final, verse des salaires $\left(w_{t} L_{t}\right)$, avec $w_{t}$ le taux de salaire nominal, et achète des biens d'équipement aux prix . Aussi, l'entreprise doit s'acquitter d'un impôt proportionnel , sur les profits.

L'objectif de la firme est de maximiser sa valeur après impôt définie comme la somme actualisée des recettes nettes que son activité procure à chaque période. Elle se présente de la façon suivante si l'horizon de la firme est infini:

$$
V=\frac{(1 \quad)\left[\begin{array}{lll}
p_{t} Y_{t} & w_{t} L_{t} & c_{t} K_{t}
\end{array}\right]}{\left(1+i_{h}\right)^{t}}
$$

où en sus des notations précédentes, $i_{h}$ est le taux d'intérêt nominal de la période $\mathrm{h} ;$, le taux d'imposition sur les bénéfices ; $Y_{t}$, la fonction de production ; $c_{t}$, le coût d'usage du capital. Ce coût ${ }^{2}$ intègre le prix du capital et les effets de l'actualisation et de la fiscalité. $c_{t}$ est défini de la façon suivante :

${ }^{2}$ Le coût d'usage du capital ou prix implicite du capital (dans la terminologie de Malinvaud (1971) représente le coût d'une unité de capital. Le stock d'actifs physiques est détenu sur longue période et l'écriture d'un prix de location pour une période revient à supposer que la firme est en mesure de revendre son capital productif à chaque fin de période. Cela suppose l'existence d'un marché concurrentiel des biens d'équipement d'occasion, à l'instar de l'approche de Jorgenson (1963). 


$$
c_{t}=\frac{p_{I, t}}{p_{t}}\left[s R+\left(\begin{array}{ll}
1 & s
\end{array}\right) \frac{I}{1}+\quad I\left(\frac{1}{1}\right)\right]
$$

avec :

- $p_{I, t}$, le prix relatif des biens d'équipement;

- $p_{t}$, le prix du bien final ;

- $s R$, les charges de la dette privée avec $R$ le taux d'intérêt nominal ;

- $\quad$, le rendement des fonds propres ;

- , le taux d'imposition ;

- , le taux de dépréciation des biens d'équipement ;

- I, l'inflation, affecté d'un facteur multiplicatif qui permet de tenir compte des distorsions liées à l'amortissement fiscal ;

- $f$, la valeur actualisée totale de l'amortissement pour un nouvel investissement de 1 franc.

Une telle définition du coût d'usage du capital appelle à au moins deux intuitions. La première a trait au passif de l'entreprise composé à la fois de fonds propres et de dettes. Le coût du capital est donc une combinaison linéaire des coûts des deux sources de financement, la pondération étant égale à leur part respective dans le bilan. La seconde se réfère à l'augmentation du taux de l'impôt sur le revenu du capital qui réduit les incitations à investir voire à innover.

Etant donné l'évolution du stock de capital :

$$
I_{t}=K_{t+1} \quad(1 \quad) K_{t}
$$

avec :

$I$, l'investissement brut ;

$K$, le stock de capital ;

$K_{t}^{*+1}$, l'investissement de remplacement à la période où , le taux de déprécation du capital à chaque période.

La firme maximise sa valeur $V$ sous les contraintes des équations [1] et [4]. Une condition $\left(K_{0}\right)$ et une condition de solvabilité assurant qu'elle honorera toujours ses dettes. Il s'agit donc de résoudre le programme intertemporel suivant :

$$
\underset{K_{t}, L_{t}}{\operatorname{Max}}\left\{V=\sum_{t=0}^{\infty} \frac{(1-\tau)\left[p_{t} Y_{t}-w_{t} L_{t}-c_{t} K_{t}\right]}{\left(1+i_{h}\right)^{t}}\right.
$$

A l'aide de la méthode de Lagrange, la résolution du programme de maximisation de la firme permet de déterminer la condition de premier ordre relative à $K_{t}$ et $L_{t}$ de la production tel que: 


$$
\begin{aligned}
& K_{t}={ }_{0} \frac{c_{t} \overline{1}}{p_{t}} \frac{w_{t}}{p_{t}} \div \\
& L_{t}={ }_{1} \frac{c_{t}}{p_{t}} \div \frac{w_{t}}{p_{t}} \div
\end{aligned}
$$

où ${ }_{0}$ et ${ }_{1}$ sont des paramètres positifs.

Quant à l'agent public, pour financer l'investissement, il prélève des taxes proportionnelles au revenu. Nous déduisons sa contrainte budgétaire à partir de celle de la firme représentative (équation [1] et [4]) :

$$
y_{t}=g_{t}^{c}+g_{t}^{p}
$$

où en sus des notations précédentes $g_{t}^{c}$ représente les dépenses publiques

improductives de la période t tel que $g_{t}^{c}=r_{t} b_{t}$ avec $r_{t}$ le taux d'intérêt réel et $b_{t}$ l'emprunt public.

Une telle présentation de la contrainte budgétaire inter-temporelle du gouvernement permet de montrer le recours aux recettes fiscales pour la réalisation des dépenses publiques appelle à au moins trois commentaires. D'abord, elle permet d'examiner l'incidence des taux de prélèvement sur la croissance via le financement des dépenses publiques productives.

Ensuite, étant donné que l'impôt provient soit du revenu des personnes physiques, soit du commerce extérieur, soit du salaire, soit du bénéfice des sociétés sa variation influence nécessairement les investissements et la croissance. A ce titre, Ferede \& Dahlby (2012) ont suggéré qu'empiriquement, une réduction de 1 point de pourcentage du taux d'imposition des sociétés est liée à une augmentation de $0,1-0,2$ point de pourcentage du taux de croissance annuel.

Enfin, les dépenses publiques improductives, sous forme de paiements d'intérêts sur la dette publique, exercent un effet d'éviction sur les dépenses productives dans la contrainte budgétaire du gouvernement.

\section{La présentation des variables du modèle}

L'analyse de la structure de la fiscalité soulève plusieurs problèmes méthodologiques dont deux au moins méritent d'être indiqués. Il y a, d'une part, la difficulté de disposer des données statistiques sur une longue période et sur les variables de politiques fiscales pour les pays concernés par l'étude. Cette difficulté a conduit à l'utilisation de « World Development Indicators » (WDI) de la Banque Mondiale comme principale source de données. D’autre 
part, il y a aussi la difficulté de trouver les statistiques qui soient suffisamment homogènes et fiables, pour permettre de faire des comparaisons inter-Etats du fait de l'instabilité socio-politique que beaucoup des pays concernés par cette étude ont connu.

Ainsi, en fonction de la disponibilité des données, nous avons construit des données de panel sur la période 1970-2016, soit sur 46 années pour 6 pays de l'Uemoa, soit un total de 276 observations.

Les variables de notre modèle sont les suivantes :

La variable expliquée du modèle est le taux de croissance du produit intérieur brut réel (y) dont la variation positive est signe de croissance

économique de l'économie considérée. La variable exogène est l'impôt sur les bénéfices des sociétés.

Les variables de contrôle comprennent l'impôt sur le revenu des personnes physiques; la taxe sur commerce extérieur ; l'impôt sur les salaires et l'impôt sur les biens et services. D’après la littérature économique, les signes attendus des variables fiscales seraient positifs mais peuvent devenir négatifs à partir d'un certain niveau.

\section{Méthode empirique}

Nous considérons une relation de non linéarité entre les variables fiscales et la croissance économique. Les modèles à changement de régime sont les plus appropriés à rendre compte de non linéarité de la dynamique des agrégats macroéconomiques. Nous proposons donc un modèle PSTR fondé sur la composition de la fiscalité. Au moins deux raisons permettent de justifier un tel choix. Premièrement, le modèle PSTR offre une possibilité intéressante de capturer l'hétérogénéité dans les données de panel et deuxièmement, il sépare clairement les observations en plusieurs ensembles ou groupes en fonction de la valeur de la variable de seuil (González, Teräsvirta \& Van Dijk, 2017).

Nous construisons donc, à différence de Ferede \& Dahlby (2012), un modèle de croissance endogène à la Barro (1990) avec l'impôt sur les bénéfices des sociétés comme source de la non-linéarité.

Le modèle fait apparaître deux régimes. Le premier concerne un impact positif de l'IBS sur la croissance. Le second se réfère à un effet négatif sur la croissance pour un niveau élevé de l'IBS. Cette non linéarité est testée par la méthode d'estimation des effets de seuils avec transition lisse en panel (PSTR) développée par González, Teräsvirta \& Van Dijk (2005). Leur démarche se présente comme suite :

Le processus $\left(Y_{i t}, \mathrm{t} \quad \mathrm{Z}\right.$ et i $\quad$ Z) satisfait une représentation PSTR à deux régimes, si et seulement si: Avec $y$, la variable expliquée, $x$, 
l'explicative et $q$ la variable de transition, l'effet de $x$ sur $y$, conditionné par $q$, peut-être estimé par le modèle Panel PSTR suivant :

$$
y_{i t}={ }_{i}+{ }_{0} x_{i t}+{ }_{1} x_{i t} G\left(q_{i t} ;, c\right)+{ }_{i t}
$$

où ${ }_{i}$ est le vecteur des effets fixes individuels et $G\left(q_{i i} ;, c\right)$, la fonction de transition associée à une variable de transition $q_{i t}$, à un paramètre de seuil $c$ et un paramètre de lissage .

Le paramètre de lissage ( )permet de caractériser à la fois la transition entre deux régimes et de conclure à l'existence ou non de non linéarités, gouvernées par $q$, entre $x$ et $y \cdot x_{i t}=\left(x_{i t}^{1}, \ldots x_{i t}^{k}\right)$ est la matrice de variables explicatives ne contenant pas de variables endogènes retardées, $=\left({ }_{1}, \ldots,{ }_{k}\right)$ et où, ${ }_{i t}$ est $i i d\left(0 ;{ }^{2}\right)$. L'indice $i=(1, \ldots, N)$ désigne la dimension individuelle et l'indice $t=(1, \ldots, T)$ la dimension temporelle.

Ainsi, en appelant (ibs, irpp, ice, is) les variables explicatives pour la variable dépendante "croissance économique" $(Y)$, la forme fonctionnelle du modèle se présente comme suite :

$$
y_{i t}=\left(i b s_{i t}, \text { irpp }_{i t}, i c e_{i t}, i s_{i t}\right) \text {, }
$$

avec :

- $y_{i t}$, la croissance du produit intérieur brut à la période $\mathrm{t}$;

- $i b s_{i t}$, l'impôt sur le bénéfice des sociétés à la période $\mathrm{t}$;

- irpp $p_{i t}$, l'impôt sur le revenu des personnes physiques à la période $\mathrm{t}$;

- ice $_{i t}$, l'impôt sur le commerce extérieur à la période $\mathrm{t}$;

$-i s_{i t}$, l'impôt sur les salaires à la période $\mathrm{t}$.

Le modèle à des fins d'estimation est le suivant :

$$
y_{i t}={ }_{i}+{ }_{0} i b s_{i t}+{ }_{1} i b s_{i t} h\left(i b s_{i t} ;, c\right)+{ }_{2} i c e_{i t}+{ }_{4} i s_{i t}+{ }_{i t} \text {, }
$$

où, en sus des notations précédentes, ${ }_{i}$ est le vecteur des effets fixes individuels $; h\left(i b s_{i t} ;, c\right)$, la fonction de transition associée à une variable de transition $i b s_{i t}$, à un paramètre de seuil et un paramètre de lissage. $c=\left(c_{1}, \ldots, c_{m}\right)$ est un vecteur de dimension $c=(1, m)$ regroupant les paramètres de seuils ; représente le paramètre de lissage supposé positif et $=\left({ }_{1}, \ldots,{ }_{k}\right)$, les élasticités du modèle.

Le modèle PSTR s'estime en quatre étapes. La première consiste à tester le modèle linéaire $(H 0: r=0)$ contre un modèle à effets de seuils avec un 
minimum de nombre de fonctions de transition à admettre dans le modèle. La seconde étape concerne la détermination du nombre de fonction de transition possible d'une manière itérative.

La procédure s'arrête quand l'hypothèse alternative est rejetée $(H 0: r=i)$ versus $(H 1: r=i+1)$ avec $(i=1, \ldots, r)$. Dans la troisième étape, nous déterminons le nombre de seuils $(m)$. Enfin, dans la dernière étape, nous estimons les paramètres du modèle PSTR par les moindres carrés non linéaires. Ces différentes étapes peuvent être regroupées en trois points que nous présentons ci-dessous.

\section{$\left.1^{\circ}\right)$ Tests de stationnarité et de linéarité}

Pour ce qui est du contrôle de la non-stationnarité des variables, nous avons utilisé le taux de croissance des variables sauf pour la variable seuil afin de faciliter l'interprétation économique.

S'agissant du test de linéarité, il a pour objectif de prouver si l'effet de seuils est statistiquement significatif et de montrer que la relation liant la variable explicative à la variable expliquée peut être représentée à l'aide d'un modèle à changements de régimes. Si le coefficient !1 est significatif, on accepte la présence d'effets non linéaire de l'impôt sur le bénéfice des sociétés $(i b s)$.

$2^{\circ}$ ) Tests de détermination du nombre et de l'ordre de fonction de transition

La détermination du nombre de fonctions de transition $(r)$ et l'ordre $(m)$ de chaque fonction de transition permet d'appréhender au mieux la relation non-linéaire entre les variables d'intérêt. Pour ce faire, la démarche suivante a été adoptée.

Tout d'abord, nous testons un modèle linéaire $(r=0)$ contre un modèle à une fonction de transition $(r=1)$. Ce test permet de vérifier la significativité de l'effet de seuil du point de vue statistique. Si le seuil est significatif, cela suppose que la relation entre la dette publique et la croissance du PIB potentiel peut-être modélisée par un modèle à changement de régime. Lorsque l'hypothèse nulle $(r=0)$ est rejetée, le modèle doit comporter au moins une fonction de transition $(r=1)$.

Nous testons ensuite l'hypothèse d'un modèle à une fonction de transition $(r=1)$ contre un modèle à deux fonctions de transition $(r=2)$. Chaque test est réalisé avec une fonction de transition d'ordre un $(m=1)$ et d'ordre deux $(m=2)$

Il est possible de tester un modèle à 3 fonctions de transition et plus avec des ordres supérieurs à deux $(m ! 2)$. Mais, lorsque le nombre de paramètres à estimer augmente, le degré de liberté des tests diminue et réduit 
la précision des tests. Aussi, faut-il un nombre minimum d'observations dans chaque régime pour garantir la crédibilité des estimations. Pour toutes ces raisons, nous nous sommes limites à deux fonctions de transition et deux seuils comme le recommande González, Teräsvirta et Van Dijk (2005).

$\left.3^{\circ}\right)$ L'estimation des paramètres du modèle PSTR par les moindres carrés non linéaires (Equation [11]).

\section{Présentation et interprétation des résultats}

Les résultats sont présentés en trois points. Le premier concerne les tests de spécification, le second porte sur l'estimation du modèle PSTR et enfin, le troisième porte sur le test de robustesse en vue de vérifier la robustesse des résultats obtenus.

\section{Tests de spécification}

Les tests de spécification comprennent l'analyse de la stationnarité des variables qui montre que toutes les variables sont stationnaires à niveau, les tests de linéarité (Tableau 1), les tests de détermination du nombre de fonction de transition et de l'ordre de la fonction de transition (Tableau 2).

La spécification d'un PSTR nécessite la détermination du nombre de fonctions de transition (r) et l'ordre (m) de chacune d'elle afin d'appréhender au mieux la relation non-linéaire entre les variables d'intérêt. Pour ce faire, la démarche suivante a été adoptée :

D'abord, nous testons un modèle linéaire $(\mathrm{r}=0)$ contre un modèle à une fonction de transition $(\mathrm{r}=1)$. Ce test permet de vérifier la significativité de l'effet de seuil du point de vue statistique. Si le seuil est significatif, cela suppose que la relation entre l'impôt sur le bénéfice des sociétés et la croissance du PIB peut-être modélisée par un modèle à changement de régime. Lorsque l'hypothèse nulle $(\mathrm{r}=0)$ est rejetée, le modèle doit comporter au moins une fonction de transition $(\mathrm{r}=1)$.

Ensuite, nous testons par la suite l'hypothèse d'un modèle à une fonction de transition $(\mathrm{r}=1)$ contre un modèle à deux fonctions de transition $(\mathrm{r}=2)$. Chaque test est réalisé avec une fonction de transition d'ordre un $(\mathrm{m}=1)$ et d'ordre deux $(\mathrm{m}=2)$. Il est possible de tester un modèle à 3 fonctions de transition et plus avec des ordres supérieurs à deux $(m>2)$. Mais, lorsque le nombre de paramètres à estimer augmente, le degré de liberté des tests diminue et réduit la précision des tests.

Aussi, faut-il un nombre minimum d'observations dans chaque régime pour garantir la crédibilité des estimations. Pour toutes ces raisons, nous nous sommes limités à deux fonctions de transition et deux seuils comme le recommande González, Teräsvirta \& Van Dijk (2005). D’ailleurs pour la dernière spécification $(\mathrm{r}=2$ et $\mathrm{m}=2)$ le nombre de points dans chaque régime 
était insuffisant pour cette estimation. Les résultats des tests de linéarité sont consignés dans le tableau suivant.

Tableau 1 : Résultat du test de linéarité

\begin{tabular}{|l|l|l|l|l|l|}
\hline Variables & \multirow{2}{*}{ Tests } & \multicolumn{2}{|l|}{ H0: $\mathrm{r}=0$ vs H1: $\mathrm{r}=1$} & \multicolumn{2}{l|}{ H0: $\mathrm{r}=1$ vs H1: $=2$} \\
\cline { 3 - 6 } & & $\mathrm{m}=1$ & $\mathrm{~m}=2$ & $\mathrm{~m}=1$ & $\mathrm{~m}=2$ \\
\cline { 2 - 6 } & Wald (LM) & $4,775(0,028)$ & $4,775(0,028)$ & $2,289(0,042)$ & - \\
\cline { 2 - 6 } & Fischer (F) & $4,542(0,033)$ & $4,542(0,033)$ & $2,123(0,046)$ & - \\
\cline { 2 - 6 } & Pseudo LRT & $4,804(0,028)$ & $4,804(0,028)$ & $2,745(0,038)$ & - \\
\hline
\end{tabular}

Source : Auteurs à partir du logiciel RATS

Note: Les valeurs entre parenthèses (.) sont les p-value ; (-) signifie absence de résultats parce que le nombre de points est insuffisant pour l'estimation de cette spécification.

Les résultats montrent que l'hypothèse de linéarité est rejetée pour deux fonctions de transition. Cependant, on remarque que le rejet de la linéarité est plus significatif pour les spécifications avec une fonction de transition. Donc nous avons choisi cette spécification. Les statistiques des tests de linéarité pour une fonction de transition sont identiques quel que soit l'ordre de la fonction de transition.

Pour effectuer un choix entre une spécification avec un seuil et une spécification avec deux seuils, nous avons recouru à d'autres critères résumés dans le tableau 2 ci-dessous. Au vu de ce tableau, la spécification avec un seul seuil possède le critère AIC, le critère de Schwarz et l'erreur d'estimation les plus faibles.

Tableau 2 : Détermination de l'ordre de la fonction de transition

\begin{tabular}{|l|l|l|l|l|}
\hline Variables & Seuils & Critère AIC & Critère de Schwarz & Erreur d'estimation \\
\hline \multirow{2}{*}{ Log (Tcpib) } & $\mathrm{m}=1$ & $-6,278$ & $-6,198$ & 0,042 \\
\cline { 2 - 5 } & $\mathrm{m}=2$ & $-6,273$ & $-6,183$ & 0,043 \\
\hline
\end{tabular}

Source : Auteurs à partir du logiciel RATS.

\section{Estimation du modèle PSTR}

Pour obtenir les résultats, nous avons exécuté le programme GTVD.SRC ${ }^{3}$ exécutable sous RATS. Le résumé des résultats est reporté dans le tableau 3 ci-dessous et le résultat complet de l'estimation est en annexe 2.

Tableau 3 : Résultats des estimations

\begin{tabular}{|l|c|c|c|}
\hline \multicolumn{1}{|c|}{ Variables } & Coefficients & Ecart type & P-Value \\
\hline Beta 0 $(\square 0)$ & 0,569 & 0,378 & 0,133 \\
\hline Beta 1 $(\square 1)$ & $-1,013 * * *$ & 0,226 & 0,000 \\
\hline Gama $(\square)$ & $752,963 * * *$ & 124,393 & 0,000 \\
\hline
\end{tabular}

${ }^{3}$ Pour plus de détails le lecteur pourrait se référer à GTVD : A RATS (Regression Analysis of Times Series) procedure to estimate Smooth Transition Regressions with Panel Data. G. Colletaz Laboratoire d'Economie d'Orléans, Université d'Orléans, France email: gilbert.colletaz@ univ-orleans.fr 


\begin{tabular}{|l|c|c|c|}
\hline Seuil (C) & $0,127 * * *$ & 0,015 & 0,000 \\
\hline Revenu des personnes physiques & $-0,233$ & 0,634 & 0,712 \\
\hline Commerce extérieur & $0,709^{* * *}$ & 0,224 & 0,001 \\
\hline Salaires & 0,283 & 0,632 & 0,654 \\
\hline Biens et services & 0,067 & 0,251 & 0,787 \\
\hline Centered R^2 & 0,085 & & \\
\hline R-Bar^2 & 0,073 & & \\
\hline Nb. Obs & 399 & & \\
\hline Nb. Pays & 19 & \multicolumn{2}{|c|}{ P-value $=0,000$} \\
\hline F Fisher F(5,393) & 7,349 & & \\
\hline
\end{tabular}

Source : Auteurs à partir du logiciel RATS. Note : *** désignent une significativité à 1\%

Les résultats de l'estimation nous permettent d'analyser l'influence du niveau de l'imposition des bénéfices des sociétés retardé d'une période sur la relation entre imposition des bénéfices des sociétés et l'activité économique par les signes de coefficient 0 et 1 .

Le coefficient 0 est positif $(0,569)$. Ce qui suppose que l'imposition des bénéfices des sociétés influence positivement l'économie lorsque l'imposition des bénéfices des sociétés retardée d'une période est inférieure à $12,7 \%$. Dans ce cas, la politique fiscale est efficace. Par contre, pour un niveau d'imposition des bénéfices des sociétés supérieur au seuil critique, l'impact de la politique d'imposition des bénéfices des sociétés est négatif $(-1,013)$. L'intuition de ce résultat est que l'imposition du bénéfice des sociétés procure dans un premier temps des ressources nécessaires au financement des dépenses publiques productives favorables à la croissance.

En effet, les recettes tirées de l'impôt sur le bénéfice des sociétés peuvent servir à l'embauche de fonctionnaires (ce qui entraîne une hausse de l'emploi), d'une part et renforcer la confiance des investisseurs extérieurs dans la stabilité et la capacité du pays à régler ses dettes, d'autre part.

En ce qui concerne les variables de contrôle, les signes des coefficients sont contraires à nos attentes. Par contre, les effets de ces variables sur le potentiel de production des pays choisis ne sont pas significatifs à l'exception de l'impôt sur le commerce extérieur. La non significativité des effets des variables de contrôle suppose qu'elles n'influencent pas directement le potentiel de production bien que n'ayant pas les signes attendus. Du point de vue théorique, les résultats sont conformes à la vision qui soutient que l'efficacité de la politique fiscale est tributaire du niveau d'imposition.

\section{Test de robustesse}

Afin de vérifier la robustesse de nos résultats, nous avons conduit des régressions en variables instrumentales pour approcher la question de l'endogénéité de nos variables d'intérêts, nous avons estimé un DMC (doubles moindres carrés). 
En effet, l'endogénéité des variables explicatives rend les coefficients estimés non convergents. La littérature économique identifie trois principales sources d'endogénéité, à savoir les erreurs de mesure des variables explicatives, la causalité réciproque entre variables dépendantes et variables exogènes et les omissions de variables explicatives majeures dans la régression.

La méthode DMC consiste à régresser la variable supposée endogène sur des variables exogènes au modèle et fortement corrélées à cette dernière. Ces régresseurs appelés instruments permettent de capter la composante exogène de la variable explicative et réaliser des estimations sans biais.

Nous avons considéré comme variables endogènes nos variables d'intérêt à savoir le taux d'imposition des bénéfices des sociétés (ibs), le taux d'imposition des bénéfices des sociétés retardée une période (ibs_1) et l'interaction imposition des bénéfices des sociétés et imposition des bénéfices des sociétés retardée d'une période (ibs_interac) qui permet d'apprécier l'effet de l'imposition des bénéfices des sociétés sur l'efficacité de la politique fiscale. Les instruments utilisés sont les retards de l'imposition des bénéfices des sociétés d'ordre 2 et 3 et le logarithme du taux de croissance d'ordre 1 à 3.

Nous avons effectué d'abord le test d'Hausman qui nous a conduit à choisir le modèle à effets aléatoires au lieu de celui à effets fixe. A la fin de l'estimation, les tests de Sargan \& Hansen sont ensuite réalisés pour tester la fiabilité des résultats. Ainsi, les résultats de ces tests montrent que l'hypothèse nulle de validité des instruments est acceptée. Ces résultats sont cohérents avec les estimations du PSTR.

On note que l'imposition des bénéfices des sociétés affecte positivement mais non significativement l'efficacité de la croissance économique. Mais, quand il est associé à l'imposition des bénéfices des sociétés retardée d'une période (ibs_interac) son effet devient significativement négatif.

Les résultats des estimations DMC sont dans l'ensemble cohérents avec l'hypothèse soutenue tout au long de cette étude. A savoir que le niveau d'imposition des bénéfices des sociétés est susceptible de réduire l'efficacité de la politique fiscale et ainsi, nuire à la croissance économique.

Tableau 4: Résultats des estimations DMC

\begin{tabular}{|l|c|c|c|}
\hline \multicolumn{1}{|c|}{ Variables } & Coefficients & $\begin{array}{c}\text { Ecart } \\
\text { type }\end{array}$ & P-Value \\
\hline Bénéfices des sociétés & 3,205 & 3,088 & 0,299 \\
\hline Bénéfices des sociétés (-1) & $4,682^{* * *}$ & 2,051 & 0,022 \\
\hline Bénéfices des sociétés interaction & $-58,759 * * *$ & 26,716 & 0,028 \\
\hline Revenu des personnes physiques & $3,734 * * *$ & 1,545 & 0,016 \\
\hline Commerce extérieur & 0,202 & 241 & 0,402 \\
\hline Salaires & $-2,331 * * *$ & 1,063 & 0,028 \\
\hline
\end{tabular}




\begin{tabular}{|l|c|l|l|}
\hline Biens et services & $-0,383$ & 0,466 & 0,411 \\
\hline Constant & $4,494 * *$ & 0,067 & 0,000 \\
\hline Anderson canon & 6,59 & $\begin{array}{l}\text { Chi-sq(3) P- } \\
\text { val=0,086 }\end{array}$ \\
\hline Cragg-Donald Wald F statistic & 1,305 & \\
\hline Sargent statistic & 0,28 & $\begin{array}{l}\text { Chi-sq(2) P- } \\
\text { val=0,869 }\end{array}$ \\
\hline
\end{tabular}

Source : Auteurs à partir dulogiciel RATS. Note : *** indiquent $\mathrm{p}<0,01$.

\section{Conclusion et implication des résultats en termes de politique économique}

Cet article a permis de déterminer, sur la base d'un modèle de croissance endogène, l'impact du niveau d'imposition du bénéfice des sociétés sur la croissance pour six (6) pays de l'Union Economique et Monétaire Ouest Africaine (UEMOA) pour la période 1970-2016. Nous avons montré qu'il ne faudrait pas nier l'ambivalence des taux d'imposition sur la croissance. Plus précisément, nous démontrons que l'imposition des bénéfices des sociétés influence positivement l'économie lorsque le taux d'imposition retardé d'une période est inférieur à $12,7 \%$. Dans ce cas, la politique fiscale est efficace. Par contre, lorsque le niveau d'imposition des bénéfices des sociétés est supérieur au seuil critique, l'impact de la politique fiscale sur la croissance devient négatif.

Un tel résultat appelle à au moins deux types de commentaire, à savoir la promotion des taux d'imposition comme moteur de l'investissement et une mauvaise politique fiscale susceptible de détruire l'économie.

$\left.1^{\circ}\right)$ Les taux d'imposition comme moteur de l'investissement

Certes la baisse des impôts sur les entreprises pourrait se traduire par une hausse des investissements. Mais, sans demande pour leurs produits, les entreprises n'investiront pas même pour un taux d'imposition proche de zéro (Stiglitz, 2016). Ainsi, en favorisant l'embauche de fonctionnaires et donc la consommation des biens et services, les recettes fiscales permettent aussi au gouvernement d'agir en tant que consommateurs de biens et services.

$\left.2^{\circ}\right)$ Une mauvaise politique fiscale peut accélérer la destruction de l'économie.

Une fiscalité lourde est susceptible de décourager le travail et l'épargne. En effet, elle réduit les capacités des entreprises à créer des emplois, d'une part et affecte le revenu que les consommateurs peuvent utiliser pour acheter des biens et des services, entraînant ainsi une perte d'activité. En conséquence, les recettes fiscales risquent fort de diminuer.

Compte tenu de ce qui précède, nous recommandons la mise en place d'un bon "système fiscal", c'est-à-dire système fiscal moins distorsif, disproportionné, ciblé et démocratique.

1- Un bon système fiscal doit être moins distorsif (Villieu, 2011), c'est-àdire qu'il ne doit pas introduire dans l'économie plus de distorsions que 
nécessaires. Ainsi, les taxes doivent davantage porter sur les biens pour lesquels leurs effets sur la demande ou sur l'offre sont limités.

2- Un bon système fiscal doit être disproportionné (OCDE, 2009) en pesant davantage sur les riches que sur les pauvres ou encore sur les entreprises qui créent moins d'emplois que sur celles qui en créent plus.

3- Il doit être bien ciblé (Bova, Kinda, Woo \& Zhang, 2013) afin de restituer l'économie. Il s'agit par exemple de stimuler les énergies renouvelables et de décourager les activités polluantes.

4- Enfin, un bon système fiscal doit apparaitre comme le produit d'un consensus démocratique (Stiglitz, 2016) et non point imposé par l'autorité.

\section{References:}

1. Avom, D. (2011). Les politiques fiscales en Afrique Centrale : une analyse empirique. Canadian Journal of Development Studies, 32:3, p.239-253.

2. Barro Robert, J. (1990). Government Spending in a Simple Model of Economic Growt. Journal of Political Economy, n98, p.103-125.

3. Barro Robert, J. et Redlick, C. J. (2011). Macroeconomic effects from government purchases and taxes. Quarterly Journal of Economics, 126 (1), p.51-102.

4. Barro Robert J., et Sala-i-Martin, X. (1992). Convergence. Journal of Political Economy, vol.100, n², p.223-251.

5. Bova, E., Kinda, T., Woo, J. et Zhang, S. (2013). Distributional Consequences of Fiscal Consolidation and the Role of Fiscal Policy : What Do the Data Say? Cahier de recherche, IMF.

6. Dowling, G. (2014). The curious case of corporate tax avoidance : is it socially irresponsible? Journal of Business Ethics, 124 (1), p.173- 184.

7. Duncan, D. et Peter, K. S. (2012). Tax Progressivity and Income Inequality. IZA DP, $\mathrm{n}^{\circ} 6910$.

8. Ferede, E. et Dahlby, B. (2012). The impact of tax cuts on economic growth: Evidence from the Canadian provinces. National Tax Journal, 65 (3), p.563-594.

9. Gemmel, N., Kneller, R. et Sanz, I. (2011). The timing and persistence of fiscal policy impacts on growth: Evidence from OECD countries. Economic Journal, 121 (550), p.F33-F58.

10. Giménez, G. A. (2015). La fiscalité et son impact négatif sur les activités d'investissement des entreprises. Note économique de l'Institut Economique Molinari. (IEM), Novembre 2015.

11. Gonzalez, A., Teräsvirta, T., Van Dijk, D. (2005). Panel Smooth Transition Regression model. SSE/EFI Working Paper Series in Economics and Finance, 604, Stockholm School of Economics. 
12. Gonzalez, A., Teräsvirta, T., Van Dijk, D. (2017). Panel Smooth Transition Regression model. CREATES Research Paper 2017-36.

13. Jaimovich, N., et Rebelo, S. (2017). « Non-Linear Effects of Taxation and Growth » Journal of Political Economy, vol.125, n¹, p.265-291.

14. Keho, Y. (2010). Effets macroéconomiques de la politique fiscale en Côte d'Ivoire. Bulletin de Politique Economique et Développement (BUPED) $\mathrm{N}^{\circ} 03 / 2010$, Cellule d'analyse de politiques 2 conomiques $\mathrm{du}$ CIRES, Côte d'Ivoire.

15. Knuutinen, R. (2014). Corporate social responsibility, taxation and aggressive tax planning. Nordic Tax Journal, 2014(1), 36-75. Retrieved from https://www.djoef-forlag.dk/sites/ntj/.

16. Mertens, K. et Morten, O. R. (2011). Empirical evidence on the aggregate effects of anticipated and unanticipated us tax policy shocks. American Economic Review, 4 (2), pp.145-181.

17. Minea, Aet Villieu, P (2011). Dette publique, croissance et bien-être : une perspective de long terme. Document de Recherche, $\mathrm{n}^{\circ}$ 2011-11, Laboratoire d'Economie d'Orléans

18. OCDE (2009). Chapitre 5. Fiscalité et croissance économique. Réformes économiques, Vol. 1, n5, p.146-168.

19. Oura, K. R. (2012). Quand l'informel prend le pas sur le formel en période de crise: le cas de Bonoua (Côte d'Ivoire). Márgenes Espacio Arte Sociedad, vol.9, n¹1, p.144-152.

20. Piketty, T., Saez, E., et Stantcheva, S. (2014). Optimal Taxation of Top Labor Incomes : A Tale of Three Elasticities. American Economic Journal : Econ. Policy, vol.6, n 1, p.230-271.

21. Romer, C. D. et Romer, D. H. (2010). The macroeconomic effects of tax changes: Estimates based on a new measure of fiscal shocks. American Economic Review, vol.100, n³, june, p.763-801.

22. Sioncke. Y. (2017). Fiscalité et innovation. Thèse de doctorat en Droit public et droit fiscal dans le cadre de École doctorale de droit de la Sorbonne (Paris).

23. Stiglitz, E. J. (2016) L'Euro : Comment la monnaie unique menace l'avenir de l'Europe, Les liens qui libèrent.

24. Stoilova, D. (2017). Tax structure and economic growth: Evidence from the European Union. Contaduría y Administración vol.62, p.1019-1040.

25. Villieu, P. (2011). Quel objectif pour la dette publique à moyen terme? Revue d'économie financière/ les politiques de sortie de crise, $\mathrm{n}^{\circ} 103$, p.79-98. 